\title{
Editorischer Bericht der Bandherausgeber
}

Die editorischen Grundsätze für die V. Abteilung (Briefwechsel und biographische Dokumente) in ihrer Modifikation ab Band 10 sind oben beschrieben. $\mathrm{Zu}$ den Einzelheiten der textkritischen Edition der Briefe vgl. die oben abgedruckten editorischen Grundsätze. Zur Orientierung der Leserin und des Lesers sei an dieser Stelle daran erinnert, dass Texte (und eben auch Briefe) zu Beginn des 19. Jahrhunderts keiner Rechtschreibnorm unterlagen und dass sich in freier schriftlicher Rede manche Ungereimtheit und mancher grammatische Fehler einschleicht, die nicht auf eine mangelhafte Transkription zurückzuführen sind. Der Editor und die Editorin des Textbandes KGA V/13 haben nur dann in Form von Textanmerkungen eingegriffen, wenn sich bei solchen Unregelmäßigkeiten Verständnisprobleme ergeben hätten. Auch die Archivierung von Briefen unterliegt keiner Norm, so dass Paginierungen, die nicht von Archivaren vorgenommen wurden, auch nicht nachträglich gesetzt werden können.

Zur besseren Orientierung und zur ersten Erschließung der Texte bieten die Textbände:

a. eine Liste der Briefe in chronologischer Sortierung,

b. ein Verzeichnis der Korrespondenz nach Korrespondenzpartnern und -partnerinnen, verbunden mit einem Überblick über deren biographische Eckdaten sowie eine Kurzcharakterisierung der Korrespondenz vom 1.7.1813 bis 31.12.1816.

Es folgen:

c. die überlieferten und erschlossenen Briefe in chronologischer Folge in textkritischer Form unter Angabe der Anmerkungen zur Überlieferung und Erstdruck sowie Anmerkungen zur Datierung und Erschließung der Briefe und gegebenenfalls deren Beilagen,

d. ein Abkürzungsverzeichnis sowie

e. ein Verzeichnis der Archive der Briefmanuskripte.

f. eine Literaturliste mit den in den Druckhinweisen und in den Anmerkungen zum Brief zitierten bibliographischen Angaben.

In dem hier relevanten Zeitraum von Juli 1813 bis Ende Dezember 1816 nimmt Schleiermacher viele amtliche Funktionen in Kirche und Staat wahr: Er führt seine Professur der Theologie an der 1810 neugegründeten 
Universität in Berlin fort, auch wenn in den Kriegsjabren 1813 und 1814 nur wenig Studierende vor Ort sind und Schleiermacher selbst einige Zeit mit dem Gedanken spielt, sich als Feldprediger in den Dienst der Armee zu stellen. Ab dem Wintersemester 1813/14 wird er für ein Jabr zum zweiten Mal Dekan der Theologischen Fakultät, ab dem Wintersemester 1815/16 für ein Jahr sogar Rektor der Universität. An der Königlich Preußischen Akademie der Wissenschaften ist er seit 1810 Mitglied der Philosophischen Klasse, nimmt an deren Sitzungen teil und hält öffentliche Akademievorträge. Im Jahr 1814 wird er zudem zum Sekretar der Philosophischen Klasse gewählt. Im Prozess der preußischen Bildungsreform, in der Schleiermacher seit 1810 auch offizielle Funktionen übernahm, wirkt er bis Anfang 1815 als ordentliches Mitglied in der Unterrichtsabteilung des Innenministeriums. Seine Pfarrstelle an der Dreifaltigkeitskirche in Berlin, die er seit dem Sommer 1809 ausfüllt, führt ebenfalls zablreiche amtliche Geschäfte mit sich. So nimmt er beispielsweise im 1811 eingerichteten Kirchenvorstandskollegium der Dreifaltigkeitskirche eine führende Rolle ein. Dieses hat mancherlei Querelen mit dem Organisten Bach zur Folge; nach dessen Tod regelt Schleiermacher mit Hilfe Zelters die Nachfolge.

Schleiermachers Einsatz als Redakteur des „Preußischen Correspondenten" ist von kurzer Dauer (vom 1. Juli bis zum 30. September 1813), fordert jedoch seine ganze Energie und zieht auch die Aufmerksamkeit der Zensur auf ihn. Die Auseinandersetzung mit Le Coq und Hardenberg erlebt Schleiermacher als ausgesprochen mühsam; er muss sich im Sommer 1814 auf einer sechswöchigen Badereise mit seiner Frau von den gesundheitlichen Auswirkungen des anstrengenden Jahres 1813 erholen.

Im Rahmen all dieser unterschiedlichen Funktionen kommt es nicht nur zu vielen neuen persönlichen Kontakten, die auch der Briefwechsel dokumentiert, sondern auch zu einer sehr umfangreichen administrativen Korrespondenz.

Bei der Sichtung der Akten im Rabmen der amtlichen Tätigkeit Schleiermachers für eine eventuelle Aufnabme in die KGA-Briefausgabe zeigte sich, dass die Gattung Brief innerhalb der Voten, Mitteilungen, Konzepte, Umlaufakten und Begleitschreiben kaum exakt zu bestimmen ist. Schreiben der einzelnen Institutionen wurden von verschiedenen Mitgliedern konzipiert, gingen in den Umlauf, wurden dann von den Mitgliedern oder einem Schreiber abgeschrieben, wobei sie zum Teil nur die Unterschrift der Institution tragen, manchmal jedoch auch noch einzelne Namen ausweisen. Schleiermacher schrieb also selbst Briefkonzepte und Notizen, er kommentierte Entwürfe, zeichnete sie gegen, er unterschrieb oder war als 
Stellvertreter und Verantwortungsträger im Namen der Institution indirekt präsent. Bei der Auswabl der für die KGA-Abteilung V aufzunehmenden Briefe sind die Herausgeber restriktiv vorgegangen: Nicht aufgenommen wurden amtliche Schreiben, die an Schleiermacher in amtlicher Funktion (z.B. als Rektor der Fakultät) gerichtet oder von ibm in amtlicher Funktion verfasst wurden, es sei denn sie enthalten auch persönliche Bezüge oder eine deutliche persönliche Stellungnahme. Eine umfassende Sichtung und Publikation der amtlichen Korrespondenz Friedrich Schleiermachers ist im Rahmen des gegenwärtigen Vorhabens nicht möglich. Die hier gebotenen amtlichen Schreiben vermitteln einen exemplarischen Eindruck seiner Tätigkeit. Weiteres Material bieten z.B. Andreas Reichs Dissertation „Friedrich Schleiermacher als Pfarrer an der Berliner Dreifaltigkeitskirche" (Schleiermacher-Archiv 12, 1992), die von Christiane Ehrhardt edierten Voten Schleiermachers zur preußischen Bildungspolitik (enthalten in KGA II/12) und die im Rahmen unseres Vorhabens im Netz publizierten Tageskalender auf der digitalen Editionsplattform „schleiermacher digital" (https://schleiermacher-digital.de/).

Sämtliche nachträglich aufgefundene und erschlossene Briefe werden in einem Ergänzungsband der Briefausgabe aufgenommen, der nach Abschluss der regulären Briefedition alle Nachträge für die gesamte Briefedition vereinigt.

Wir danken allen Personen und Institutionen, die uns bei der Bearbeitung des Bandes unterstützt haben: Die Abteilung Telota an der Berlin-Brandenburgischen Akademie der Wissenschaften mit Nadine Arndt, Stefan Dumont, Martin Fechner, Lou Klappenbach und Frederike Neuber hat für die Schleiermacherforschungsstelle eine XML-basierte Datenbank erarbeitet und in stetem Austausch mit uns immer weiter verbessert; sie hat die Entstehung des Bandes bis zur Drucklegung betreut. Zu danken ist Prof. Dr. Andreas Arndt, Projekt- und langjähriger Arbeitsstellenleiter des Vorhabens und Dr. Wolfgang Virmond ( + ), die an den Vorarbeiten zu dem vorliegenden Band wesentlichen Anteil hatten; sie und unsere Kollegin Elisabeth Blumrich und unser Kollege Dr. Holden Kelm haben uns auch in vielen Einzelfragen weitergeholfen. Für die Fragen binsichtlich der Manuskript-Bestände im Universitätsarchiv Krakau stand uns dankbarerweise Prof. Dr. Piotr Bukowski zur Seite. Isabelle Lüke, die langjährige wissenschaftlich-technische Mitarbeiterin der Forschungsstelle, Johann Gartlinger als studentische Hilfskraft und Felix Will als Praktikant haben die Fertigstellung des Bandes ebenfalls mit großem Engagement unterstützt, auch ihnen möchten wir herzlich danken. Auch Dr. Albrecht Döhnert und Lena Hummel sowie Stefan Diezmann 
XL

Editorischer Bericht der Bandherausgeber

vom Verlag de Gruyter sei der beste Dank für ibre Unterstützung während der Herstellung ausgesprochen. Und schließlich sei Prof. Dr. Lutz Käppel als geschäftsfübrendem Herausgeber der KGA Schleiermachers für seine Ratschläge und Hinweise herzlich gedankt.

Berlin, im Februar 2020

Simon Gerber \& Sarah Schmidt 